Cahiers $d u$ MONDE RUSSE

\section{Cahiers du monde russe}

Russie - Empire russe - Union soviétique et États indépendants

$56 / 4 \mid 2015$

Médiateurs d'empire en Asie centrale (1820-1928)

\title{
J. Arch Getty, Practicing Stalinism, Bolsheviks, Boyars, and the Persistence of Tradition
}

\section{Yves Cohen}

\section{OpenEdition}

\section{Journals}

Édition électronique

URL : http://journals.openedition.org/monderusse/8257

DOI : $10.4000 /$ monderusse. 8257

ISSN : $1777-5388$

\section{Éditeur}

Éditions de l'EHESS

\section{Édition imprimée}

Date de publication : 1 octobre 2015

Pagination : 857-861

ISBN : 978-2-7132-2507-9

ISSN : $1252-6576$

Référence électronique

Yves Cohen, «J. Arch Getty, Practicing Stalinism, Bolsheviks, Boyars, and the Persistence of Tradition », Cahiers du monde russe [En ligne], 56/4 | 2015, mis en ligne le 01 octobre 2015, Consulté le 24 septembre 2020. URL : http://journals.openedition.org/monderusse/8257 ; DOI : https://doi.org/ 10.4000/monderusse.8257

Ce document a été généré automatiquement le 24 septembre 2020. 


\title{
J. Arch Getty, Practicing Stalinism, Bolsheviks, Boyars, and the Persistence of Tradition
}

\author{
Yves Cohen
}

\section{RÉFÉRENCE}

J. ARCH GETTY, Practicing Stalinism, Bolsheviks, Boyars, and the Persistence of Tradition, Yale :Yale University Press, 2013, 384 p.

Ce livre est surprenant et décevant.

2 Arch Getty, professeur de l'université de Californie à Los Angeles, est un historien important de l'Union soviétique. Son ouvrage de 1985, Origins of the Great Purges, a profondément marqué le débat historiographique tel qu'il se déployait à l'époque entre les «totalitariens » partisans d'une histoire politique et les " révisionnistes » partisans d'une histoire sociale. A. Getty a été l'un des principaux auteurs du second pôle puis un explorateur actif des nouvelles archives des années 1990. L'ouvrage recensé était attendu. On savait l'auteur mobiliser son immense connaissance des sources soviétiques pour établir de façon définitive que le pouvoir stalinien était intégralement fondé sur des relations personnelles et l'on se réjouissait d'avance de sa publication. L'auteur nous montre " la personnalisation de la politique » avec une grande richesse d'exemples extraits des archives des années 1920 et 1930. Ce ne sont pas la modernité ni la rationalité affichées, ni non plus le marxisme-léninisme, qui dictent les décisions, mais les liens personnels. L'ouvrage étudie la montée du patrimonialisme et des privilèges oligarchiques. Boyards, satrapes, clans et « familles » sont les formes sociales selon lesquelles se forme le pouvoir. Mais la question vient vite : est-ce bien cet ouvrage que nous livre Arch Getty ? Est-ce seulement cela que l'auteur cherche à nous dire ? Après tout, ces thèses sont familières et d'ailleurs, ce 
qu'il ne dit pas, le jeu entre les personnes et les formes institutionnelles au bénéfice des premières n'est en rien propre à la Russie, qu'elle soit soviétique ou éternelle.

Selon l'auteur, il conduit le lecteur non pas dans la fiction moderniste mais dans la réalité des " pratiques ", " la vie réelle ». Or nous ne lisons pas de véritable étude de pratique. L'auteur reconnaît d'ailleurs opérer une " sélection éclectique » de faits que sa longue fréquentation des archives lui permet de prélever surtout dans des fonds centraux. Le talent narratif d'A. Getty rend la lecture toujours agréable. Pourtant, il ne s'installe nulle part pour observer, sur une période de quelques mois ou de quelques années, à l'aide de sources confrontées les unes aux autres, ce que font les acteurs qui l'intéressent en quelque ville ou administration, à l'occasion de quelque événement ou au prétexte de quelque processus. Il aurait pu le faire tout aussi bien au centre que, par exemple, dans le district de Iaroslav qui est l'un des mieux documentés, mais toujours à partir de sources non locales. En fait, la sélection des sources et des faits est ad hoc et jamais contradictoire : ils doivent convenir à la thèse défendue. Ceci constitue le premier des quatre principaux problèmes de méthode que pose cet ouvrage.

4 Pratiquer le stalinisme n'aide pas à élaborer ce que pourrait être une histoire des pratiques dans le monde russe et soviétique. Ce livre au fond cherche-t-il même à parler des pratiques? C'est de structures qu'Arch Getty nous entretient, de "structures profondes", qu'il appelle encore des "racines profondes". Ces structures proviennent du passé et elles orientent les gestes du présent. La pratique politique du « système personnalisé » est incrustée (embedded) dans la culture russe, qui n'a donc pas d'histoire, mais une essence. Elle constitue un " héritage inconscient ", même pour les vieux bolcheviks : "Ils en étaient des héritiers, sinon des prisonniers ", " no choice ». Arch Getty recourt à Pierre Bourdieu pour prouver que la tradition peut agir les personnes à leur insu. Rien de neuf depuis Rjurik, et son héritage porte jusqu'à aujourd'hui : "Dans la mesure où nous trouvons cette conception de la politique du gouvernement à tous les niveaux des hiérarchies, en tout temps et en tous lieux, depuis Rjurik jusqu'à Putin, il était impossible d'y échapper. Il s'agit de la structure profonde par laquelle la Russie a toujours été gouvernée ». Que reste-t-il d'un historien à celui qui s'institue l'interprète exclusif d'une réalité inaccessible au commun des mortels et considérée comme un inconscient ? Nous sommes loin d'une recherche comme enquête patiente pour rouvrir les possibles sans cesse refermés par la dynamique historique. Toute histoire est écrasée sous ce long millénaire initié par un grand acte fondateur et qui ne cesse de s'entretenir. Notons au passage que le sous-titre comporte "the Persistence of Tradition " sans que soit évoqué le livre classique d'Arno Mayer sur La Persistance de l'Ancien Régime en Europe (New York, 1981; Paris, 1983) qui aurait pu ancrer l'ouvrage dans un débat historiographique. Nous n'avons pas d'étude des pratiques en acte, en train de se faire. La négation des formes institutionnelles ne nous aide pas à comprendre leur rôle ni la débauche d'efforts dépensés pour les concevoir et les mettre en place, alors que l'historiographie récente regorge d'hypothèses. Considérés depuis les pratiques, ces efforts en sont une dimension et ils sont le fait de personnes engagées dans leurs pratiques, aussi personnelles soient-elles.

5 L'auteur reprend ensuite à nouveaux frais le dossier qui l'a occupé dès les années 1980, celui des purges. L'un des passages les plus forts de l'ouvrage montre les « petits Stalin » locaux s'opposer ouvertement à la perspective de candidatures multiples aux élections des Soviets qui sont prévues par la première version de la «Constitution 
démocratique " de 1936, et plus tard à celles du parti. La grande terreur des années 1937 et 1938 aurait été lancée par un Stalin désireux de soutenir la volonté des clans locaux d'éliminer toute opposition putative. L'idée d'une purge généralisée viendrait d'en bas et suivrait un modèle conçu par S.N. Mironov, chef du NKVD de Sibérie occidentale. Il aurait été transformé et étendu à toute l'Union soviétique par "l'ordre » central du NKVD n 447, désormais fort connu, qui visait les koulaks et tous les opposants au régime soviétique et qui a eu pour conséquence la plus grande partie des arrestations et des exécutions sans procès de ces années : « Il semble clair [it seems clear] que la décision de Stalin d'approuver l'opération de masse provienne des craintes locales envers l'opposition dans les campagnes, déclenchée et légitimée par la nouvelle constitution si impopulaire parmi les barons locaux ». Voici à quoi se résume ce que l'ouvrage propose sur la Grande Terreur et voici aussi, deuxième problème de méthode, l'un de ses principaux oublis opportuns. En effet, outre cet Ordre 447, ont été lancées durant le même été 1937 plusieurs opérations et en particulier des "opérations nationales " visant les personnes relevant de toute une série de "nationalités ", principalement celles vivant dans des zones frontalières, Allemands, Polonais, Coréens, Lettons, Finlandais, etc. Si la terrible répression de ces années était susceptible de procéder en partie de la volonté d'aller au-devant du désir répressif d'élites régionales qui seraient, au demeurant, détruites dans la foulée, ce ne serait que parmi bien d'autres raisons.

6 Thèse importante, A. Getty indique que rien ne prouve que la répression (réduite donc à l'ordre 447) soit conçue comme une " mesure prophylactique " prise dans la crainte de la guerre. Il appelle à l'aide Nikolaj Buharin qui, dans sa dernière lettre à Stalin, le 10 décembre 1937, avant d'être exécuté, le félicite pour la " grande et audacieuse idée de purge générale ». A. Getty rapporte que Buharin « écrit » " qu'une purge générale était en partie connectée au "passage à la démocratie" "(p.235). Cette dernière expression citée par l'auteur vise à confirmer que l'anticipation d'une guerre n'entrait pas en ligne de compte. Mais pourquoi tronquer le propos de Buharin ? La phrase entière se lit en effet comme suit : " Il y a la grande et audacieuse idée de purge générale a) en relation avec la menace de guerre, b) en relation avec le passage à la démocratie ${ }^{1}$. " Le passage non reproduit par A. Getty et désigné par son expression " en partie » est en effet gênant pour sa thèse, même s'il n'est pas à lui seul susceptible de l'invalider (Buharin écrit au pied de l'échafaud quand Stalin est au Kremlin, et ce sont les raisons de ce dernier que l'on cherche).

7 En tout cas, troisième problème de méthode, une source a été tronquée pour les besoins de la cause. S'il reste une question sur le rapport de Stalin au meurtre de masse, elle ne saurait se régler de cette façon. L'opération est censée donner l'image d'un Stalin poussé au meurtre de masse par les subordonnés qu'il a lui-même choisis dans les régions et les républiques, qui forment non pas des clans séparés mais des filiales du sien propre, et dont il se débarrasse avec aussi peu d'émotion que de ses ennemis déclarés, s'il en avait encore.

8 Il semble important pourtant de noter encore un autre procédé car il est une composante de la leçon que nous offre cet ouvrage. Les « familles » des « féodaux » ou " seigneurs » débusqués par Stalin (après qu'il les a eu lui-même sélectionnés et promus) sont l'objet d'une mobilisation de tout le parti et des organes de répression à partir du plénum de février 1937. L'ouvrage montre à partir de nouvelles sources combien ces clans étaient consistants, coupés des masses (le vocabulaire du 
marxisme-léninisme étant endossé pour l'occasion), et combien régnait le favoritisme. $\mathrm{Au}$ plénum lui-même et dans d'autres assemblées de ce type dans les régions et républiques montent les accusations de la base. Or celles-ci sont prises pour argent comptant, dans leur facticité affichée, sans étude circonstanciée et contradictoire de leurs conditions de production ni des épisodes évoqués par les orateurs. Aucune précaution d'usage n'est mentionnée : l'information vient d'en bas, elle dénonce les " familles ", elle est donc vraie. Là s'observe la méthode d'étude des pratiques développée par l'auteur. La présentation critique des extraits choisis fait défaut. Aucune confrontation des sources n'est menée ni de vérification locale des dires de ces cadres. Or ceux-ci s'expriment sous de violents projecteurs dans une atmosphère de terreur palpable dans laquelle nul ne sait qui est susceptible d'être visé. Un des procédés de défense est bien indiqué : il consiste à retourner l'accusation sur qui vous accuse. Mais un autre est ignoré, qui court tout au long de l'histoire du stalinisme, et qui consiste à accuser l'accusé, à participer à la curée pour mieux s'en protéger. Ces propos de plénums et de conférences participent certainement en partie à ce procédé mais rien n'est vérifié, leur vérité n'est pas assurée : la seule chose que l'on sache est qu'ils ont été prononcés.

Sur le fond, sans préjuger de tout ce qui reste à dire, on s'interroge sur l'intérêt pour la discipline historique de confier la causalité à un inconscient collectif et plus que millénaire qui, d'une part, renvoie à des théories de psychanalyse, de psychologie et de psychologie sociale très discutées et non pas à l'histoire et, d'autre part, institue l'historien en psychanalyste des peuples. Ce livre souffre donc de profonds défauts méthodologiques : il apprend premièrement à sélectionner les sources et les événements qui conviennent à une thèse préétablie, deuxièmement à pratiquer d'opportuns oublis de données qui pourraient gêner ladite thèse, troisièmement à faire parler des sources sans référence à leur production et sans enquête contradictoire et, last but not least, à tronquer des documents en y supprimant ce qui serait embarrassant pour la thèse en question.

\section{NOTES}

1. Nicolas Boukharine, «Lettre à Staline " (10 décembre 1937), Bulletin de l'IHTP, $\mathrm{n}^{\circ} 73$, mars 1999 , p. 16 (soulignement de NB). 


\section{AUTEURS}

\section{YVES COHEN}

École des Hautes Études en Sciences Sociales 